\title{
HIGH RESOLUTION LIDAR-DERIVED ELEVATION DATA FOR BARRY ARM LANDSLIDE, SOUTHCENTRAL ALASKA, JUNE 26, 2020
}

Ronald P. Daanen, Gabriel J. Wolken, Katreen Wikstrom Jones, and Andrew M. Herbst

Raw Data File 2021-3

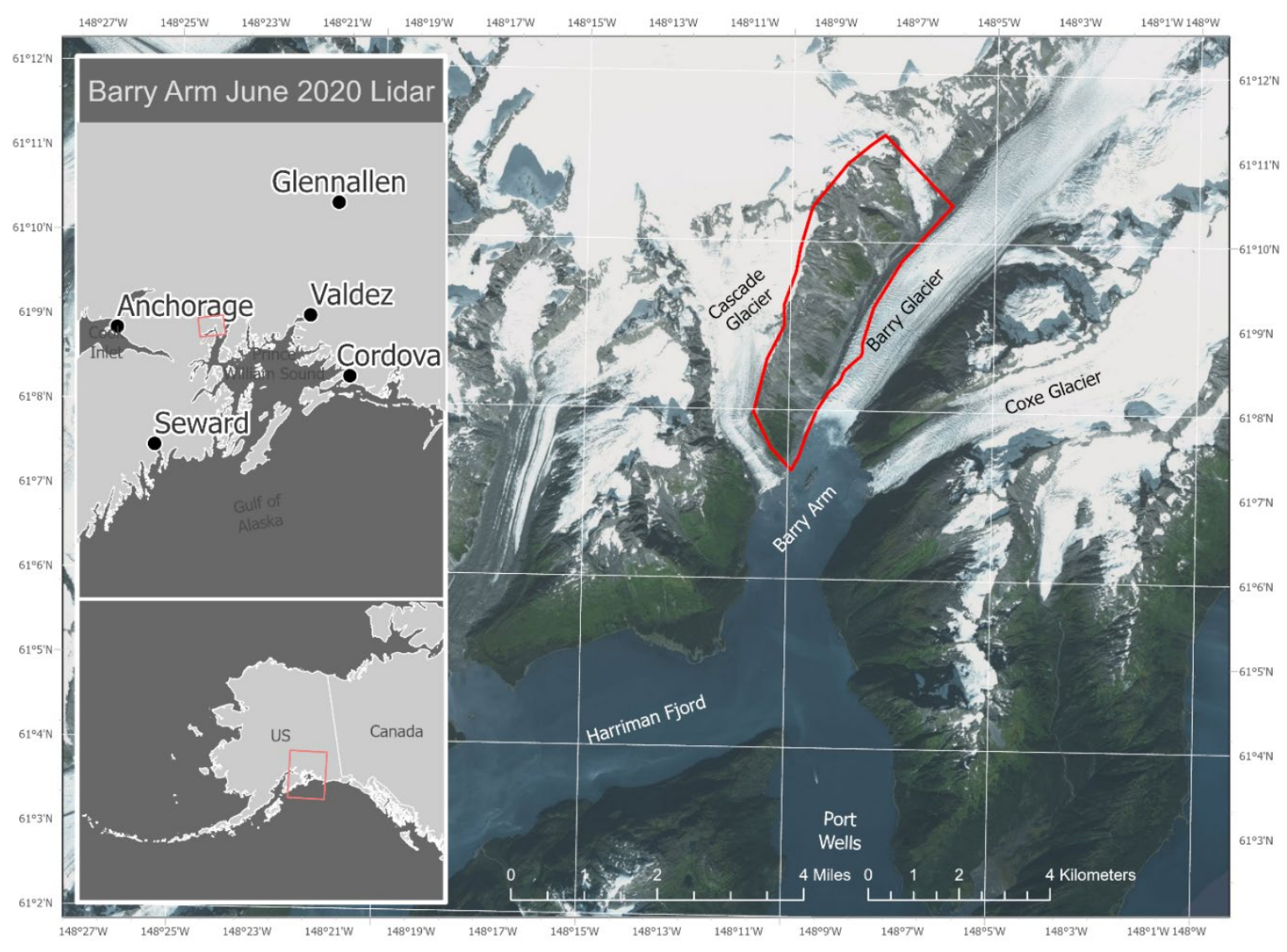

Location map of focused area of interest with orthometric image.

This report has not been reviewed for technical content or for conformity to the editorial standards of DGGS.

2021

STATE OF ALASKA

DEPARTMENT OF NATURAL RESOURCES

DIVISION OF GEOLOGICAL \& GEOPHYSICAL SURVEYS

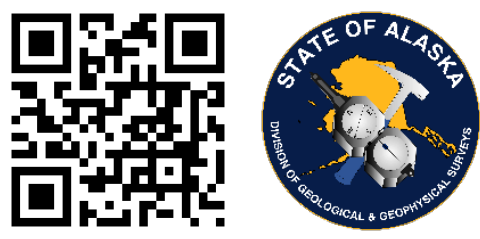


STATE OF ALASKA

Mike Dunleavy, Governor

DEPARTMENT OF NATURAL RESOURCES

Corri A. Feige, Commissioner

\section{DIVISION OF GEOLOGICAL \& GEOPHYSICAL SURVEYS}

Steve Masterman, State Geologist \& Director

Publications produced by the Division of Geological \& Geophysical Surveys are available to download from the DGGS website (dggs.alaska.gov). Publications on hard-copy or digital media can be examined or purchased in the Fairbanks office:

\section{Alaska Division of Geological \& Geophysical Surveys (DGGS)}

3354 College Road | Fairbanks, Alaska 99709-3707

Phone: 907.451.5010 | Fax 907.451.5050

dggspubs@alaska.gov | dggs.alaska.gov

DGGS publications are also available at:

Alaska State Library, Historical

Collections \& Talking Book Center

395 Whittier Street

Juneau, Alaska 99801

Alaska Resource Library and

Information Services (ARLIS)

3150 C Street, Suite 100

Anchorage, Alaska 99503

\section{Suggested citation:}

Daanen, R.P., Wolken, G.J., Wikstrom Jones, Katreen, and Herbst, A.M., 2021, High resolution lidar-derived elevation lidar data for Barry Arm landslide, Southcentral Alaska, June 26, 2020: Alaska Division of

Geological \& Geophysical Surveys Raw Data File 2021-3, 9 p.

http://doi.org/10.14509/30593
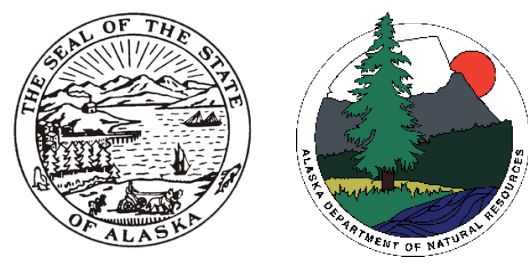


\title{
HIGH RESOLUTION LIDAR-DERIVED ELEVATION LIDAR DATA FOR BARRY ARM LANDSLIDE, SOUTHCENTRAL ALASKA, JUNE 26, 2020
}

Ronald P. Daanen ${ }^{1}$, Gabriel J. Wolken ${ }^{1}$, Katreen Wikstrom Jones ${ }^{1}$, and Andrew M. Herbst ${ }^{1}$

\section{INTRODUCTION}

The Alaska Division of Geological \& Geophysical Surveys (DGGS) used aerial lidar to produce a classified point cloud and high resolution digital terrain model (DTM), digital surface model (DSM), and intensity model of the Barry Arm landslide, northwest Prince William Sound, Alaska, during near snow-free ground conditions on June 26, 2020. The survey's goal is to provide high quality and high resolution $(0.10 \mathrm{~m})$ elevation data to assess potential landslide movement. Aerial lidar and ground control data were collected on June 26, 2020, and subsequently processed in Terrasolid and ArcGIS. Ground control was collected on June 26, 2020, as well. This data collection is released as a Raw Data File with an open end-user license. All files can be downloaded free of charge from the DGGS website: https://doi.org/10.14509/30593.

\section{LIST OF DELIVERABLES}

\author{
Classified Points \\ DSM and DTM \\ Intensity Image \\ Metadata
}

\section{MISSION PLAN}

\section{Lidar Survey Details}

DGGS used a Riegl VUX1-LR laser scanner integrated with a global navigation satellite system (GNSS) and Northrop Grumman LN-200C inertial measurement unit (IMU). Phoenix LiDAR Systems designed the lidar integration system. The sensor is capable of collecting up to 820,000 points per second over a distance of $150 \mathrm{~m}$. This survey was flown with a pulse refresh rate between 200,000 and 600,000 pulses per second at a scan rate between 80 and 150 lines per second. This survey was flown with an average elevation of $200 \mathrm{~m}$ above ground level and a ground speed of approximately $36 \mathrm{~m} / \mathrm{s}$ with a fixed-wing aircraft configuration, using a Cessna 180 aircraft. The scan angle was set from 80 to 280 degrees. The total area surveyed on the mission was approximately $40 \mathrm{~km}^{2}$ (figure 1). The focused area of interest depicted in the location map (cover page figure) is approximately $6.1 \mathrm{~km}^{2}$ and, to achieve a higher pulse density and hence a higher resolution product for this section, was flown with the highest pulse refresh rate and scan rate from the above mentioned ranges.

\footnotetext{
${ }^{1}$ Alaska Division of Geological \& Geophysical Surveys, 3354 College Road, Fairbanks, Alaska 99709
} 


\section{Weather Conditions and Flight Times}

The aerial survey was flown on June 26, 2020, with a Cessna 180. Flight take-off occurred at 10:15 am from Merrill Field airport in Anchorage, Alaska, and landing occurred at 4:00 pm. The aircraft landed at the airstrip in Girdwood, Alaska, once during the survey. The weather throughout the survey was overcast.

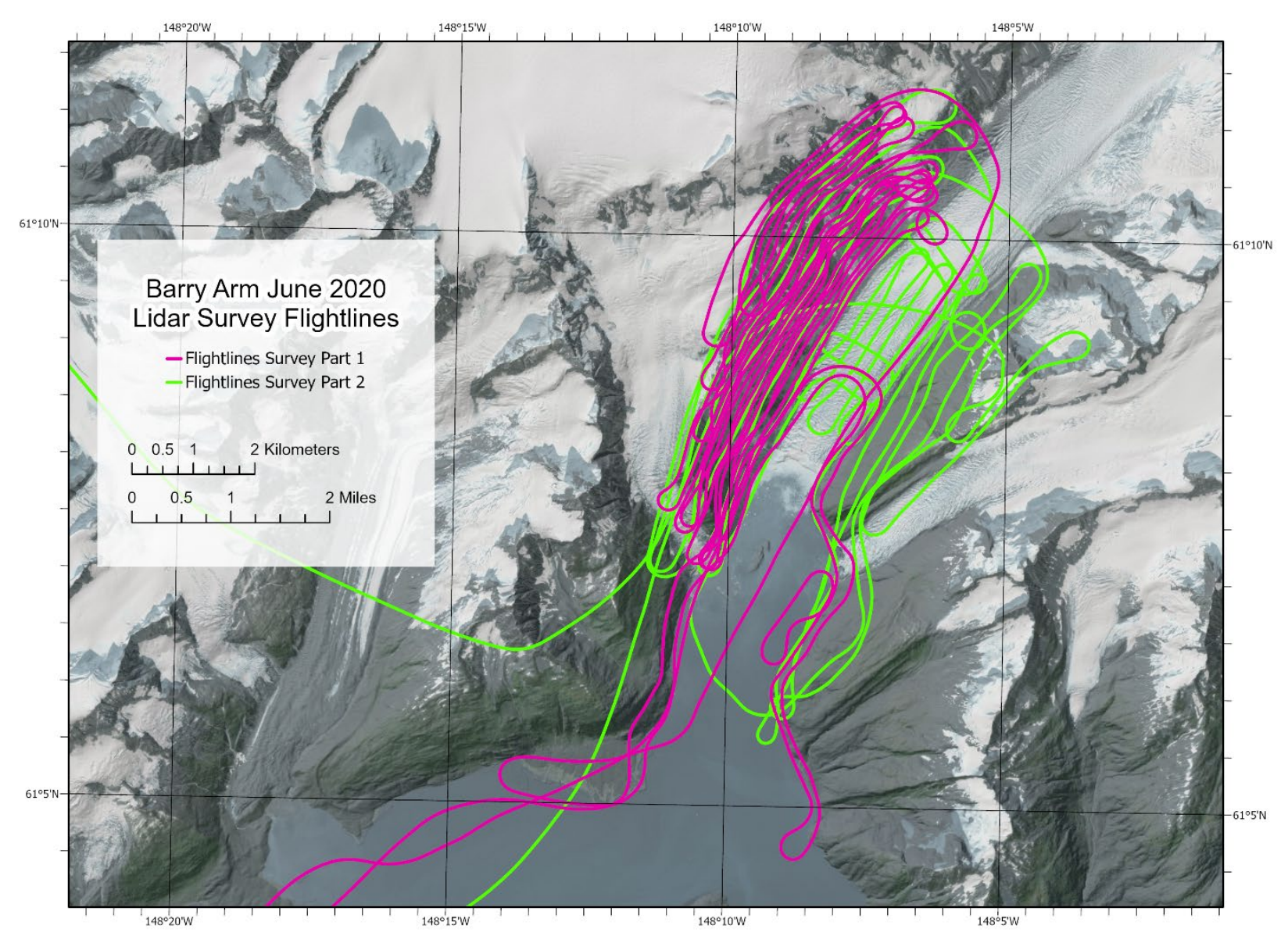

Figure 1. Project flightlines. The aircraft returned to Girdwood, Alaska, for refueling between the two parts of the survey.

\section{Lidar Dataset Processing}

Point data were processed in SDCimport software for initial filtering and multiple-timearound (MTA) disambiguation. MTA errors, corrected in this process, result from imprecise interpretations of received pulse time intervals and occur more frequently with higher pulse refresh rates. IMU and GNSS data were processed in Inertial Explorer and used to integrate flightline information with the point cloud in Spatial Explorer software. The point data were calibrated at an incrementally precise scale of sensor movement and behavior, incorporating sensor velocity, roll, pitch, and yaw fluctuations throughout the survey. 
Points were classified in accordance with American Society for Photogrammetry and Remote Sensing (ASPRS) 2014 guidelines, using macros designed in Terrasolid software. Careful attention was given to the interpolation of the project's ground surface to compensate for inconsistent penetration through low vegetation as a function of the scan angle. Once classified, points underwent a geometric transformation and were converted from ellipsoidal heights to GEOID12B (Alaska) orthometric heights.

Raster products were derived from the point cloud, using ArcMap. The DTM was interpolated from all ground class returns using a tin-based method. The DSM was interpolated from only the first return points using a tin-based method. An intensity image was also produced in ArcMap, using closest-to-mean binning.

\section{Classified Point Cloud}

Classified point cloud data is provided in this collection in compressed LAZ format. Data are classified in accordance with ASPRS 2014 guidelines and contain return and intensity information. The average pulse spacing was $1.8 \mathrm{~cm}$ and the average density was $53.8 \mathrm{pts} / \mathrm{m}^{2}$ (figure 2).

\section{Digital Surface Model}

The DSM represents surface elevations, including heights of vegetation, buildings, bridges, etc. The DSM is a single band, 32-bit GeoTIFF file, with a ground sample distance of 10 centimeter. No Data value is set to $-3.40282306074 \mathrm{e}+038$.

\section{Digital Terrain Model}

The DTM represents surface elevations of ground surfaces, excluding vegetation, bridges, buildings, etc. The DTM is a single-band, 32-bit float GeoTIFF file, with a ground sample distance of 10 centimeters. No Data value is set to $-3.40282306074 \mathrm{e}+038$.

\section{Lidar Intensity Image}

The lidar intensity image portrays the relative amplitude of reflected signals contributing to the point cloud. Lidar intensity is largely a function of scanned object reflectance in relation to the signal frequency, is dependent on ambient conditions, and is not necessarily consistent between separate scans. The intensity image is a single-band, 32-bit float GeoTIFF file with a ground sample distance of 10 centimeters. No Data value is set to $-3.40282306074 \mathrm{e}+038$ (32-bit, floating-point minimum). 


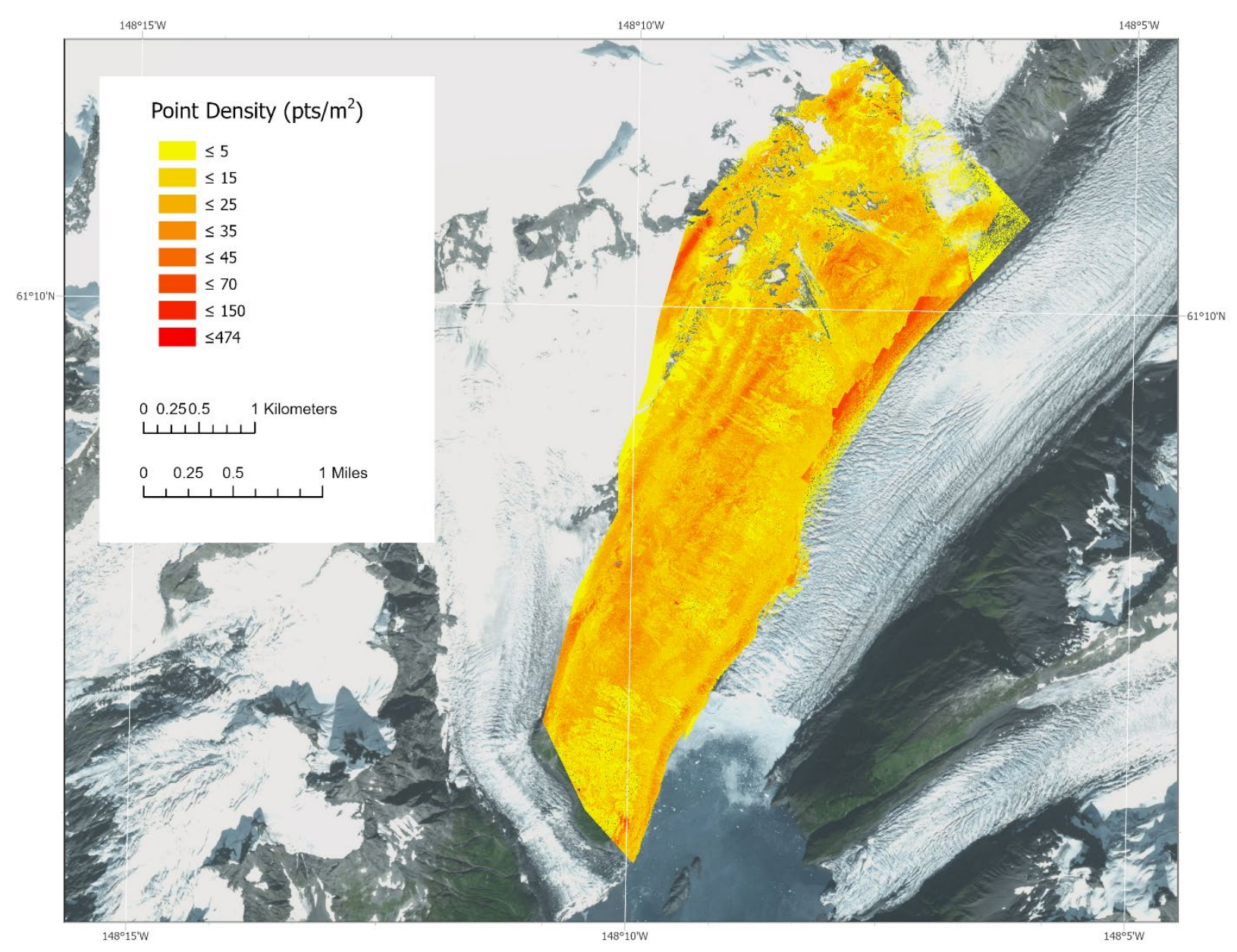

Figure 2. Ground point density for the survey displayed as a 1-meter raster.

\section{Ground Survey Details}

Ground control and check points were collected on June 26, 2020. A Trimble R10-2 GNSS receiver with internal antenna was deployed on a ridge near the center of the study area and provided a base station occupation and real-time kinematic (RTK) corrections to points surveyed with a rover Trimble R10-2 GNSS receiver (internal antenna). A total of 106 ground control points and check points were collected to be used for calibration and assessment of the vertical accuracy of the point cloud. All points were collected on bare earth or minimally vegetated surface.

\section{Coordinate system and Datum}

All data were processed and delivered in NAD83 (2011) UTM6N and vertical datum NAVD88 GEOID12B.

\section{Horizontal Accuracy}

Horizontal accuracy was not measured for this collection. 


\section{Vertical Accuracy}

A mean offset of $-19.7 \mathrm{~cm}$ was measured between 84 control points and the point cloud (appendix 1). This offset was reduced to $-0.6 \mathrm{~cm}$ by performing a vertical transformation of the lidar point data. Twenty-two check points were used to determine the non-vegetated vertical accuracy (NVA) of the point cloud ground class, using a tin-based approach. Project NVA was calculated to have a root mean square error (RMSE) of $7.7 \mathrm{~cm}$ (appendix 2). Relative accuracy for this dataset was evaluated as the interswath overlap consistency and was measured at $8.3 \mathrm{~cm}$ RMSE.

\section{Data Consistency and Completeness}

This data release is complete, and there is no over collect, except for the aircraft turns that were eliminated from the dataset. The data quality is consistent throughout the survey.

\section{ACKNOWLEDGMENTS}

These data products were funded by the State of Alaska and collected and processed by DGGS. We thank Clearwater Air and Alpine Air for their aviation expertise and contribution to these data products. 
APPENDIX 1: GROUND CONTROL POINTS

\begin{tabular}{|c|c|c|c|c|c|}
\hline Number & Easting (m) & Northing (m) & Known Z (m) & Laser Z (m) & $\mathrm{Dz}(\mathrm{m})$ \\
\hline 1 & 437591.962 & 6779373.098 & 551.72 & 551.64 & -0.08 \\
\hline 2 & 436713.426 & 6777598.259 & 576.13 & 576.05 & -0.08 \\
\hline 3 & 438635.954 & 6779406.265 & 1.15 & 1.06 & -0.09 \\
\hline 4 & 437586.279 & 6779373.005 & 551.70 & 551.58 & -0.12 \\
\hline 5 & 436698.359 & 6777611.065 & 577.39 & 577.27 & -0.12 \\
\hline 6 & 438092.254 & 6780055.176 & 534.73 & 534.60 & -0.13 \\
\hline 7 & 438025.996 & 6780010.381 & 535.88 & 535.75 & -0.13 \\
\hline 8 & 438074.328 & 6780047.293 & 535.65 & 535.51 & -0.14 \\
\hline 9 & 438110.665 & 6780074.356 & 536.90 & 536.76 & -0.14 \\
\hline 10 & 437121.962 & 6779963.546 & 1077.80 & 1077.66 & -0.14 \\
\hline 11 & 437063.569 & 6779919.568 & 1092.55 & 1092.41 & -0.14 \\
\hline 12 & 437603.353 & 6779347.912 & 546.07 & 545.93 & -0.14 \\
\hline 13 & 437053.638 & 6779969.355 & 1089.89 & 1089.74 & -0.15 \\
\hline 14 & 436659.12 & 6777643.689 & 583.41 & 583.26 & -0.15 \\
\hline 15 & 438036.659 & 6780032.136 & 539.47 & 539.32 & -0.15 \\
\hline 16 & 437059.688 & 6779908.314 & 1094.02 & 1093.87 & -0.15 \\
\hline 17 & 438578.37 & 6779430.798 & 1.07 & 0.92 & -0.15 \\
\hline 18 & 437173.064 & 6779972.558 & 1059.01 & 1058.86 & -0.15 \\
\hline 19 & 436764.743 & 6777575.569 & 581.85 & 581.70 & -0.15 \\
\hline 20 & 436782.216 & 6777582.11 & 585.16 & 585.00 & -0.16 \\
\hline 21 & 438597.602 & 6779433.641 & 1.74 & 1.58 & -0.16 \\
\hline 22 & 437102.83 & 6779929.491 & 1082.19 & 1082.03 & -0.16 \\
\hline 23 & 436738.694 & 6777566.623 & 574.90 & 574.74 & -0.16 \\
\hline 24 & 438612.821 & 6779415.983 & 1.41 & 1.25 & -0.16 \\
\hline 25 & 438600.134 & 6779425.974 & 1.38 & 1.21 & -0.17 \\
\hline 26 & 438059.556 & 6780032.257 & 534.94 & 534.77 & -0.17 \\
\hline 27 & 437596.62 & 6779333.029 & 547.19 & 547.02 & -0.17 \\
\hline 28 & 436989.148 & 6779694.531 & 1085.22 & 1085.05 & -0.17 \\
\hline 29 & 436899.865 & 6779520.404 & 1073.92 & 1073.75 & -0.17 \\
\hline 30 & 437058.217 & 6779878.001 & 1092.51 & 1092.34 & -0.17 \\
\hline 31 & 437588.839 & 6779321.496 & 548.31 & 548.14 & -0.17 \\
\hline 32 & 437152.156 & 6779922.686 & 1055.05 & 1054.87 & -0.18 \\
\hline 33 & 438049.541 & 6780033.66 & 537.88 & 537.70 & -0.18 \\
\hline 34 & 438040.893 & 6780022.431 & 535.42 & 535.23 & -0.19 \\
\hline 35 & 438739.764 & 6777118.416 & 4.42 & 4.23 & -0.19 \\
\hline 36 & 437038.714 & 6779767.095 & 1092.30 & 1092.11 & -0.19 \\
\hline 37 & 437063.29 & 6779967.066 & 1086.52 & 1086.33 & -0.19 \\
\hline 38 & 436762.539 & 6777587.52 & 582.10 & 581.91 & -0.19 \\
\hline 39 & 438809.321 & 6776426.297 & 2.19 & 2.00 & -0.19 \\
\hline
\end{tabular}




\begin{tabular}{|c|c|c|c|c|c|}
\hline Number & Easting $(\mathrm{m})$ & Northing (m) & Known Z (m) & Laser Z (m) & $\mathrm{Dz}(\mathrm{m})$ \\
\hline 40 & 437052.822 & 6779879.368 & 1092.89 & 1092.70 & -0.19 \\
\hline 41 & 438609.673 & 6779455.663 & 4.43 & 4.23 & -0.20 \\
\hline 42 & 436979.276 & 6779659.208 & 1084.79 & 1084.59 & -0.20 \\
\hline 43 & 436937.771 & 6779586.708 & 1070.21 & 1070.01 & -0.20 \\
\hline 44 & 438851.052 & 6776427.826 & 4.58 & 4.38 & -0.20 \\
\hline 45 & 437174.411 & 6779963.277 & 1059.10 & 1058.89 & -0.21 \\
\hline 46 & 438845.597 & 6776426.869 & 4.24 & 4.03 & -0.21 \\
\hline 47 & 437099.469 & 6779920.163 & 1082.69 & 1082.48 & -0.21 \\
\hline 48 & 438714.577 & 6777155.687 & 3.81 & 3.60 & -0.21 \\
\hline 49 & 437028.041 & 6779753.279 & 1091.26 & 1091.05 & -0.21 \\
\hline 50 & 438716.171 & 6777117.814 & 2.84 & 2.63 & -0.21 \\
\hline 51 & 436709.936 & 6777521.463 & 585.86 & 585.65 & -0.21 \\
\hline 52 & 437005.539 & 6779715.967 & 1085.51 & 1085.30 & -0.21 \\
\hline 53 & 436940.402 & 6779601.176 & 1071.81 & 1071.60 & -0.21 \\
\hline 54 & 436740.439 & 6777498.496 & 574.43 & 574.21 & -0.22 \\
\hline 55 & 438806.177 & 6776442.733 & 1.36 & 1.14 & -0.22 \\
\hline 56 & 438805.367 & 6776436.85 & 1.58 & 1.36 & -0.22 \\
\hline 57 & 437133.914 & 6779989.986 & 1080.46 & 1080.24 & -0.22 \\
\hline 58 & 437574.423 & 6779362.076 & 552.91 & 552.69 & -0.22 \\
\hline 59 & 438047.573 & 6780023.708 & 535.91 & 535.69 & -0.22 \\
\hline 60 & 438596.605 & 6779456.506 & 4.46 & 4.24 & -0.22 \\
\hline 61 & 437041.743 & 6779790.815 & 1087.55 & 1087.33 & -0.22 \\
\hline 62 & 436918.501 & 6779570.779 & 1070.23 & 1070.01 & -0.22 \\
\hline 63 & 436743.959 & 6777503.268 & 573.64 & 573.41 & -0.23 \\
\hline 64 & 438040.59 & 6780032.095 & 540.08 & 539.85 & -0.23 \\
\hline 65 & 436984.61 & 6779683.229 & 1084.94 & 1084.71 & -0.23 \\
\hline 66 & 436873.024 & 6779482.811 & 1073.92 & 1073.68 & -0.24 \\
\hline 67 & 437034.04 & 6779815.834 & 1097.63 & 1097.39 & -0.24 \\
\hline 68 & 438839.743 & 6776438.745 & 2.49 & 2.25 & -0.24 \\
\hline 69 & 437138.137 & 6779929.709 & 1062.37 & 1062.13 & -0.24 \\
\hline 70 & 437127.883 & 6779983.913 & 1080.05 & 1079.81 & -0.24 \\
\hline 71 & 438713.084 & 6777137.841 & 3.32 & 3.08 & -0.24 \\
\hline 72 & 437164.84 & 6779944.321 & 1061.34 & 1061.10 & -0.24 \\
\hline 73 & 437052.283 & 6779863.405 & 1093.77 & 1093.53 & -0.24 \\
\hline 74 & 436873.275 & 6779505.256 & 1081.58 & 1081.33 & -0.25 \\
\hline 75 & 436862.729 & 6779470.046 & 1071.13 & 1070.87 & -0.26 \\
\hline 76 & 438087.15 & 6780053.167 & 535.95 & 535.69 & -0.26 \\
\hline 77 & 437175.955 & 6779992.477 & 1062.17 & 1061.90 & -0.27 \\
\hline 78 & 437596.238 & 6779360.879 & 550.09 & 549.82 & -0.27 \\
\hline 79 & 436947.858 & 6779642.69 & 1080.37 & 1080.10 & -0.27 \\
\hline
\end{tabular}




\begin{tabular}{|c|c|c|c|c|c|}
\hline Number & Easting (m) & Northing (m) & Known Z (m) & Laser Z (m) & Dz (m) \\
\hline $\mathbf{8 0}$ & 437195.609 & 6780011.539 & 1065.20 & 1064.92 & -0.28 \\
\hline $\mathbf{8 1}$ & 438052.134 & 6780025.833 & 535.66 & 535.37 & -0.29 \\
\hline $\mathbf{8 2}$ & 437571.472 & 6779313.945 & 552.59 & 552.27 & -0.32 \\
\hline $\mathbf{8 3}$ & 437646.068 & 6776672.209 & 29.32 & 28.99 & -0.33 \\
\hline $\mathbf{8 4}$ & 437635.514 & 6776681.925 & 26.58 & 26.25 & -0.33 \\
\hline & & & & \\
\hline Average dz (m) & -0.197 & & & \\
\hline Minimum dz (m) & -0.332 & & & \\
\hline $\begin{array}{c}\text { Mverage magnitude error } \\
\text { (m) }\end{array}$ & -0.081 & & & \\
\hline $\begin{array}{c}\text { Root mean square error } \\
\text { (m) }\end{array}$ & 0.197 & & & \\
\hline Standard deviation (m) & 0.051 & & & \\
\hline
\end{tabular}

\section{APPENDIX 2: CHECK POINTS}

\begin{tabular}{|c|c|c|c|c|c|}
\hline Number & Easting (m) & Northing $(\mathbf{m})$ & Known Z (m) & Laser Z (m) & Dz (m) \\
\hline $\mathbf{1}$ & 438056.676 & 6780038.106 & 536.54 & 536.64 & 0.10 \\
\hline $\mathbf{2}$ & 436715.424 & 6777580.726 & 573.06 & 573.14 & 0.08 \\
\hline $\mathbf{3}$ & 437569.37 & 6779336.199 & 551.86 & 551.94 & 0.08 \\
\hline $\mathbf{4}$ & 437628.425 & 6776623.844 & 36.79 & 36.84 & 0.05 \\
\hline $\mathbf{5}$ & 437605.252 & 6779335.395 & 546.35 & 546.40 & 0.05 \\
\hline $\mathbf{6}$ & 438641.658 & 6779408.426 & 1.04 & 1.08 & 0.04 \\
\hline $\mathbf{7}$ & 437049.746 & 6779836.095 & 1097.47 & 1097.49 & 0.02 \\
\hline $\mathbf{8}$ & 437099.17 & 6779937.926 & 1082.44 & 1082.46 & 0.02 \\
\hline $\mathbf{9}$ & 437057.016 & 6779957.892 & 1087.72 & 1087.74 & 0.02 \\
\hline $\mathbf{1 0}$ & 438831.48 & 6776422.876 & 3.45 & 3.46 & 0.01 \\
\hline $\mathbf{1 1}$ & 436725.691 & 6777527.669 & 577.17 & 577.18 & 0.01 \\
\hline $\mathbf{1 2}$ & 437054.73 & 6779895.159 & 1093.69 & 1093.70 & 0.01 \\
\hline $\mathbf{1 3}$ & 437170.289 & 6779953.716 & 1060.17 & 1060.18 & 0.01 \\
\hline $\mathbf{1 4}$ & 437601.908 & 6779332.037 & 546.62 & 546.62 & 0.01 \\
\hline $\mathbf{1 5}$ & 438065.83 & 6780037.472 & 535.61 & 535.60 & -0.01 \\
\hline $\mathbf{1 6}$ & 438736.779 & 6777105.668 & 3.64 & 3.63 & -0.01 \\
\hline $\mathbf{1 7}$ & 438031.041 & 6780014.56 & 535.25 & 535.23 & -0.02 \\
\hline $\mathbf{1 8}$ & 436908.894 & 6779533.061 & 1072.59 & 1072.56 & -0.03 \\
\hline $\mathbf{1 9}$ & 437615.1 & 6776693.71 & 33.01 & 32.92 & -0.09 \\
\hline $\mathbf{2 0}$ & 436968.514 & 6779649.814 & 1084.67 & 1084.57 & -0.10 \\
\hline $\mathbf{2 1}$ & 437013.034 & 6779730.265 & 1087.85 & 1087.71 & -0.14 \\
\hline $\mathbf{2 2}$ & 437202.15 & 6780017.197 & 1067.27 & 1067.03 & -0.24 \\
\hline & & & & & \\
\hline $\mathbf{2 0}$ & & & & \\
\hline
\end{tabular}




\begin{tabular}{|c|c|c|c|c|c|}
\hline Number & Easting (m) & Northing (m) & Known Z (m) & Laser Z (m) & $D z(m)$ \\
\hline $\begin{array}{c}\text { Average } \\
d z(m)\end{array}$ & -0.006 & & & & \\
\hline $\begin{array}{l}\text { Minimum } \\
\text { dz (m) }\end{array}$ & -0.241 & & & & \\
\hline $\begin{array}{l}\text { Maximum } \\
\text { dz (m) }\end{array}$ & 0.1 & & & & \\
\hline $\begin{array}{l}\text { Average } \\
\text { magnitude } \\
\text { (m) }\end{array}$ & 0.052 & & & & \\
\hline $\begin{array}{c}\text { Root } \\
\text { mean } \\
\text { square } \\
\text { error }(m)\end{array}$ & 0.077 & & & & \\
\hline $\begin{array}{c}\text { Standard } \\
\text { deviation } \\
\text { (m) }\end{array}$ & 0.078 & & & & \\
\hline
\end{tabular}

